
\title{
$\varepsilon_{\sin }$
}

\author{
${ }^{1}$ Field Epidemiology Services- \\ Victoria, Public Health \\ England, London, UK \\ ${ }^{2}$ North Central London TB \\ Service, Royal Free Hospital \\ NHS Foundation Trust, \\ University College London, \\ London, UK \\ ${ }^{3}$ Department of Infection and \\ Population Health, Centre for \\ Infectious Disease \\ Epidemiology, University \\ College London, London, UK \\ ${ }^{4}$ Respiratory Diseases \\ Department, Centre for \\ Infectious Disease Surveillance \\ and Control, Public Health \\ England, London, UK
}

\section{Correspondence to}

Charlotte Anderson, Field Epidemiology ServicesVictoria, Public Health England, 151 Buckingham Palace Road, London SW1W 9SZ, UK;

Charlotte.anderson@phe.gov. uk

Received 23 April 2013 Revised 21 May 2013 Accepted 22 June 2013
To cite: Anderson $C$, White J, Abubakar I, et al. Thorax Published Online First: [please include Day Month Year] doi:10.1136/ thoraxjnl-2013-203751

\section{Raising standards in UK TB control: introducing cohort review}

\author{
Charlotte Anderson, ${ }^{1}$ Jacqueline White, ${ }^{2}$ Ibrahim Abubakar, ${ }^{3,4}$ Marc Lipman, ${ }^{2}$ \\ Surinder Tamne, ${ }^{4}$ Sarah R Anderson, ${ }^{1}$ Jennifer deKoningh, ${ }^{2}$ Susan Dart ${ }^{2}$
}

\section{ABSTRACT}

Cohort review has been used internationally to support tuberculosis (TB) control. We describe its first use in the UK by a London TB service. Improvements were noted in case management and contact tracing, weaknesses identified and important service changes put in place. Key areas of impact were directly observed therapy (DOT) provision (a greater proportion of cases offered DOT, and in response to low uptake resources diverted to create posts responsible for patient-centred DOT delivery), and contact tracing (more contacts per case screened and assessed). Cohort review enables whole system review and improvement. It has subsequently been adopted across the UK.

\section{INTRODUCTION}

Tuberculosis (TB) is an on-going problem in the UK. ${ }^{1}$ Unlike most other countries, rates are not falling. It is concentrated in urban areas with the greatest number in London where, in 2011, there were approximately 3500 new notifications. ${ }^{2}$ The five clinics that comprise North Central London (NCL) TB Service notify approximately 500 new cases per year. ${ }^{2}$ This is more than most UK cities and many European capitals. ${ }^{1}$

TB cases are socially complex, with one in 10 known to have associated issues of homelessness, problem drug or alcohol use, or imprisonment. ${ }^{2}$ Management is further complicated by increasing TB drug resistance. ${ }^{2}$ Having systems that are in place which routinely and effectively enable TB services to detect, treat and prevent $\mathrm{TB}$ are, therefore, of paramount importance.

In 2010, NCL TB Service introduced cohort review with the aim of improving clinical and public health TB management. Cohort review is the multidisciplinary, systematic, quarterly appraisal of the case management and contact investigation of every case of TB. It focusses on specific outcomes, measured against local and national targets, and provides a framework for service evaluation. The process was adapted from US Centers for Disease Control and Prevention guidance, ${ }^{3}$ and drew upon the success of cohort review in a number of TB control programmes, including New York City, where since its adoption in 1993, there has been a steep and progressive decline in TB rates. ${ }^{4}$

As its first UK adopter, we describe our experience, the impact of its methodology on our TB service, and implications for others.

\section{METHODS}

All patients notified as having TB disease by the five NCL TB clinics between 1 July 2009 and the implementation of cohort review in June 2010 were compared with those diagnosed in the first 18 months of its use (1 July 2010 to 31 December 2011). A standard set of information was obtained on each patient, including: treatment outcome at 12 months; offer and uptake of HIV testing; identification of the need for, and use of, directly observed therapy (DOT); and the number of contacts identified and assessed (excluding larger incident screening exercises). Data were analysed using Stata 12. The proportion of cases with the outcome of interest in each cohort were compared using $\chi^{2}$ tests (or Fisher's exact test where warranted by small cell sizes), with Bonferroni correction to control for the number of comparisons. As this was a service evaluation, ethical approval was not required.

\section{RESULTS}

Five hundred and fifty-seven TB cases were notified by NCL clinics in the year before cohort review was introduced (table 1). Over the next 18 months, 752 cases were notified. Both groups were similar in terms of sex, age, place of birth and drug resistance. Postcohort review, more of the non-UK-born cases were recent migrants (15\% vs $10 \%)$, although nearly half had still been in the UK for 10 or more years before diagnosis. Fewer cases had pulmonary disease in the post-cohort review group, while the proportion confirmed by culture increased. Fewer cases were reported as having one or more social risk factor in the postcohort review group (22\% vs $16 \%, \mathrm{p}=0.005$ ) (table 1).

We considered the impact of cohort review on two areas: (1) TB case management outcomes, including anti-TB treatment effect, uptake of HIV testing, and the use of DOT; and (2) contact tracing outcomes, including the number of contacts identified and assessed.

\section{TB case management}

Over $90 \%$ of cases were offered a HIV test, and were tested, in both groups. Excluding those with known rifampicin resistance, there was no change in the proportion of cases completing treatment following the implementation of cohort review (table 1).

A higher proportion of adult cases in the cohort review group was assessed as requiring DOT, particularly those with identified risk factors $(38 \%$ and 
Table 1 Characteristics, management and contact tracing outcomes of TB cases notified before and after cohort review implementation

\begin{tabular}{|c|c|c|c|c|c|}
\hline & \multicolumn{2}{|c|}{ Precohort review } & \multicolumn{2}{|c|}{ Postcohort review } & \multirow[b]{2}{*}{$p$ Value ${ }^{*}$} \\
\hline & $\bar{n} / \mathrm{N}$ & $\%$ & $\mathrm{n} / \mathrm{N}$ & $\%$ & \\
\hline \multicolumn{6}{|l|}{ Description of cases } \\
\hline \multicolumn{6}{|l|}{ Patient demographics } \\
\hline Male & $307 / 557$ & 55.1 & $407 / 752$ & 54.1 & 0.721 \\
\hline Aged 20-39 years & $248 / 557$ & 44.5 & $365 / 752$ & 48.5 & 0.150 \\
\hline Non-UK born & $409 / 541$ & 75.6 & $586 / 742$ & 79.0 & 0.152 \\
\hline Recent migrant ( $<2$ years) & $44 / 425$ & 10.4 & $88 / 596$ & 14.8 & 0.038 \\
\hline Long-term resident ( $\geq 10$ years) & $243 / 425$ & 57.2 & $274 / 596$ & 46.0 & $<0.001$ \\
\hline Reported risk factor on LTBRT & $120 / 547$ & 21.9 & $116 / 735$ & 15.8 & 0.005 \\
\hline \multicolumn{6}{|l|}{ Clinical characteristics } \\
\hline Pulmonary & $328 / 557$ & 58.9 & $394 / 752$ & 52.4 & 0.020 \\
\hline Sputum & $111 / 328$ & 52.3 & $139 / 394$ & 52.1 & 0.975 \\
\hline Culture confirmed & $278 / 557$ & 49.9 & $418 / 752$ & 55.6 & 0.042 \\
\hline Any drug resistance & $39 / 278$ & 14 & $54 / 418$ & 12.9 & 0.673 \\
\hline MDR & $7 / 274$ & 2.6 & 9/417 & 2.2 & 0.735 \\
\hline \multicolumn{6}{|l|}{ HIV test } \\
\hline Offered & $511 / 557$ & 91.7 & $694 / 752$ & 92.3 & 0.718 \\
\hline Performed & $510 / 557$ & 91.6 & $687 / 752$ & 91.4 & 0.895 \\
\hline \multicolumn{6}{|l|}{ Case management and contact tracing outcomes } \\
\hline \multicolumn{6}{|l|}{ Treatment outcome at 12 months $\ddagger$} \\
\hline Completed & $480 / 549$ & 87.4 & $637 / 737$ & 86.4 & 0.599 \\
\hline Still on treatment & $23 / 549$ & 4.2 & $44 / 737$ & 6.0 & 0.155 \\
\hline Died & $24 / 549$ & 4.4 & $20 / 737$ & 2.7 & 0.106 \\
\hline Lost to follow-up & $12 / 549$ & 2.2 & $25 / 737$ & 3.4 & 0.201 \\
\hline lost to follow-up overseas & $4 / 549$ & 0.7 & $16 / 737$ & 2.2 & 0.039 \\
\hline excluding those lost to follow-up overseas & $8 / 549$ & 1.5 & 9/737 & 1.2 & 0.714 \\
\hline Treatment stopped & $10 / 549$ & 1.8 & $11 / 737$ & 1.5 & 0.645 \\
\hline \multicolumn{6}{|l|}{ DOT§ } \\
\hline Required DOT & $62 / 380$ & 16.3 & $145 / 680$ & 21.3 & 0.049 \\
\hline Required DOT (with identified risk factort) & $42 / 110$ & 38.2 & $79 / 134$ & 59.0 & 0.001 \\
\hline Offered DOT & $58 / 62$ & 93.6 & $135 / 145$ & 93.1 & 0.907 \\
\hline Offered DOT (with identified risk factort) & $41 / 42$ & 97.6 & $73 / 79$ & 92.4 & 0.242 \\
\hline Received DOT & $52 / 62$ & 83.9 & $91 / 145$ & 62.8 & 0.003 \\
\hline Received DOT (with identified risk factort) & $38 / 42$ & 90.5 & $56 / 79$ & 70.9 & 0.014 \\
\hline Refused DOT & $6 / 62$ & 9.7 & $44 / 145$ & 30.3 & 0.001 \\
\hline Refused DOT (with identified risk factort) & $3 / 42$ & 7.1 & $17 / 79$ & 21.5 & 0.043 \\
\hline \multicolumn{6}{|l|}{ One or more contacts identified } \\
\hline All cases & $430 / 557$ & 77.2 & $646 / 752$ & 85.9 & $<0.001$ \\
\hline Pulmonary & $257 / 328$ & 78.4 & $345 / 394$ & 87.6 & 0.001 \\
\hline Sputum smear positive & $102 / 111$ & 91.9 & $132 / 139$ & 95.0 & 0.324 \\
\hline \multicolumn{6}{|l|}{ Three or more contacts identified } \\
\hline All cases & $282 / 557$ & 50.6 & $428 / 752$ & 56.9 & 0.024 \\
\hline Pulmonary & $179 / 328$ & 54.6 & $252 / 394$ & 64.0 & 0.010 \\
\hline Sputum smear positive & $82 / 111$ & 73.9 & $115 / 139$ & 82.7 & 0.089 \\
\hline \multicolumn{6}{|l|}{ Five or more contacts identified } \\
\hline All cases & $160 / 557$ & 28.7 & $233 / 752$ & 30.1 & 0.378 \\
\hline Pulmonary & $107 / 328$ & 32.6 & $144 / 394$ & 36.6 & 0.270 \\
\hline Sputum smear positive & $61 / 111$ & 55.0 & $75 / 139$ & 54.0 & 0.875 \\
\hline \multicolumn{6}{|l|}{ Contacts assessed } \\
\hline All cases & $1312 / 1777$ & 73.8 & $2134 / 2644$ & 80.7 & $<0.001$ \\
\hline Pulmonary & $856 / 1162$ & 73.7 & $1301 / 1594$ & 81.6 & $<0.001$ \\
\hline Sputum smear positive & $433 / 559$ & 77.5 & $621 / 735$ & 84.5 & 0.001 \\
\hline
\end{tabular}

$59 \%$ preintroduction and postintroduction, respectively, $\mathrm{p}=0.001)$. However, although the proportion offered DOT by the clinic remained high $(>90 \%)$, the proportion that actually received DOT fell. This largely resulted from an increased refusal by patients to be treated using DOT $(10-30 \%, \mathrm{p}=0.001)$ (table 1). 


\section{Contact tracing outcomes}

Following the introduction of cohort review, the proportion of cases with at least one contact identified among all cases rose $(p<0.001)$, in particular for pulmonary cases $(p=0.001)$. The proportion of cases with three or more contacts identified also increased $(55-64 \%, \mathrm{p}=0.010$ for pulmonary cases). There was, however, no evidence of a change in the proportion of TB cases with five or more contacts identified which remained at 30\% of all cases, and a third of pulmonary cases and half of sputum smear positive cases.

The proportion of contacts that were clinically assessed by the TB team increased from $74 \%$ to $81 \%(\mathrm{p}<0.001)$. Importantly, this was found in contacts of pulmonary $(74-82 \%, \mathrm{p}<0.001)$ and sputum smear positive cases $(78-85 \%, \mathrm{p}=0.001)$, who are at greatest risk of infection.

\section{DISCUSSION}

It is widely recognised that early identification of TB cases and improving treatment completion are key to TB control. ${ }^{1}$ Identification is largely through self-presentation to health services, contact tracing around known cases, and active case finding in high-risk populations. Ensuring that cases successfully complete treatment relies on the implementation of standardised case management practices, with extra support for vulnerable patients. Insufficient evidence has been available to determine the extent and success of contact tracing practices in London, and in the identification and enhanced care of patients at risk of default. This paper reviews the first use of cohort review in the UK, and its impact on a TB service.

The basic premise of cohort review is a framework of peer appraisal to ensure standardisation of case management. The systematic process highlights strengths and weaknesses of the service as a whole that may not be identified in other case review or team meetings.

We found some measures of successful case management, in particular, treatment completion and HIV testing, remained high but unchanged. As these were very high to begin with, a larger sample size would be needed to show a statistically significant effect.

After cohort review was implemented, a higher proportion of adults were assessed as requiring DOT though less received itmainly due to patient refusal. This often arises because individuals feel they do not need supervised therapy, or that DOT interferes with their schedule. ${ }^{5}$ Education, support and ensuring arrangements for DOT are person-centred, and may overcome these concerns. However, cohort review also highlighted staff concerns regarding the service's ability to provide DOT, in particular, out-of-hours, at weekends and public holidays, and across geographic service boundaries. Our solution was to identify funds to employ four non-clinical staff with the specific remit to deliver community-based DOT. We believe this will resolve many of the issues over DOT provision, and the DOT team's impact will be monitored through cohort review.

We found some evidence that a greater number of contacts were screened and assessed once cohort review was initiated. Importantly, this was performed in cases where the index was likely to be most infectious. The whole system approach, inherent in cohort review, highlighted several deficiencies in our current contact tracing practice, informed the development of new national guidance, enhanced staff development and emphasised the importance of timely and accurate data collection. Further plans include routine community visits (particularly for smear positive pulmonary patients) to identify high-risk contacts, and a new standard to visit all contacts of pulmonary $\mathrm{TB}$ cases who fail to attend two appointments for screening. A further service objective is to provide an out-of-hours contact tracing clinic to offer patients increased choice of appointment times.

A limitation of our evaluation is that we cannot account for subjective changes in the way staff assessed and managed patients that may have occurred as a result of increased focus on process and outcome. For example, while the precohort review group was reported as having a greater proportion of cases with one or more social risk factors, the proportion assessed as requiring DOT was higher in the postcohort review groupwhich may result from more stringent assessments by case managers.

Following our implementation, it has been adopted across London and the UK. We urge TB services to ensure it is used appropriately, and its impact closely monitored. For example, increasing the number of contacts screened could be a significant burden to TB services unless the focus remains on those at highest risk. A fuller evaluation of the impact of cohort review across the UK is planned.

We have described the first use of cohort review in the UK: it has proved to be an effective, practical tool to monitor patient and public health outcomes for a local TB Service. We observed an improvement in a number of case management and contact tracing outcomes, but more importantly it has allowed the service to better identify its weaknesses and find solutions to these collectively. Its use in both high and low incidence areas indicates that cohort review is an adaptable tool applicable to a variety of settings.

Contributors All authors substantially contributed to this manuscript. CA, JW and SD had the idea for the study; CA, JW, SD and IA contributed to the study design; CA and JD contributed to acquisition of data; CA and IA contributed to analysis of data; CA drafted the article; All authors revised it critically for interpretation and important intellectual content. All authors gave final approval to the version to be published. CA and SD are the guarantors.

\section{Competing interests None.}

Provenance and peer review Not commissioned; externally peer reviewed.

\section{REFERENCES}

1 Abubakar I, Lipman M, Anderson C, et al. Tuberculosis in the UK-time to regain control. BMJ 2011;343:d4281.

2 Tuberculosis in London: Annual review (2011 data). Health Protection Agency London Regional Epidemiology Unit. September 2012. http://www.hpa.org.uk/webc/ HPAwebFile/HPAweb_C/1317136048986

3 Centers for Disease Control and Prevention, Division of Tuberculosis Elimination. Understanding the TB cohort review process: instruction guide. Atlanta, GA: CDC, 2006. http://www.cdc.gov/nchstp/tb/pubs/cohort/cohort.pdf (accessed Apr 2011).

4 Munsiff SS, Ahuja SD, King L, et al. Ensuring accountability: the contribution of the cohort review method to tuberculosis control in New York City. Int J Tuberc Lung Dis 2006;10:1133-9.

5 Gupta S, Berg D, de Lott F, et al. Directly observed therapy for tuberculosis in New York City: factors associated with refusal. Int J Tuberc Lung Dis 2004:8:480-5. 